\title{
Does Schizophrenia in Offspring Increase the Risk of Developing Alzheimer's Dementia?
}

\author{
Christopher Rohde $^{a} \quad$ Esben Agerbo ${ }^{a-c}$ Philip Rising Nielsen ${ }^{a, b}$ \\ ${ }^{a}$ National Centre for Register-Based Research, Aarhus University, ${ }^{\mathrm{b}}$ Lundbeck Foundation \\ Initiative for Integrative Psychiatric Research, iPSYCH, and ${ }^{\mathrm{C} C I R R A U}-$ Centre for Integrated \\ Register-Based Research at Aarhus University, Aarhus, Denmark
}

\section{Key Words}

Dementia · Cohort study · Psychiatric disorders · Familial coaggregation · Etiology

\begin{abstract}
Background/Aims: Prior studies have consistently found a higher risk of dementia in individuals with schizophrenia, but whether this is due to a common etiology between the disorders remains obscure. We wanted to elucidate this association by investigating whether schizophrenia in offspring increases the risk of Alzheimer's dementia. Methods: All individuals born between 1930 and 1953 were identified through national registers and followed from their 50th birthday until the date of Alzheimer's dementia, death or end of the study. Regressions were performed to evaluate the association between offspring with schizophrenia and Alzheimer's dementia. Results: Individuals with offspring with schizophrenia did not have an increased risk of Alzheimer's dementia [incidence rate ratio (IRR), 0.97; 95\% CI, 0.88-1.07] compared to individuals with offspring without psychiatric contact. This finding remained stable when evaluating early-onset (IRR, 1.10; 95\% CI, 0.91-1.31) and late-onset Alzheimer's dementia (IRR, 0.92; 95\% CI, 0.88-1.07). Similar findings were made for vascular and unspecified dementia. Conclusion: The finding of no familial coaggregation between schizophrenia and Alzheimer's dementia may suggest that no common etiology between the disorders exists. This may indicate that the excess risk of dementia in individuals with schizophrenia is a by-product of the higher rates of somatic comorbidity and adverse health risk factors among these individuals.




\section{Introduction}

Dementia is one of the most frequent and serious neurodegenerative disorders in later life. It is characterized by a progressive loss of cognitive and functional abilities associated with various behavioral disturbances, which will eventually lead to debilitation and death [1, 2]. Due to increased life expectancy in most industrialized and many developing nations, a worldwide increase in the prevalence of dementia is expected $[3,4]$. This increase may challenge the health and social welfare systems, and place a substantial financial burden on society [5, 6], making dementia a major public health priority.

Despite the fact that our understanding of the neuropathology of dementia has advanced dramatically during the last 30 years [7], there is still a deficiency of an effectual therapeutic strategy to prevent disease progression [8]. Given the dearth of curative treatment, effective coping strategies towards the expected worldwide increase in dementia will have to comprehend efficient and sustainable prevention strategies. To design such strategies, there is a need for a greater appreciation of determinants and modifiable risk factors for dementia. Most studies to date have been devoted to investigate environmental and medical risk factors $[9,10]$, but it has also been suggested that dementia may partly share etiology, i.e. a common genetic or neurobiological liability or mutual risk factors, with certain severe mental disorders such as schizophrenia. This shared etiology has been motivated by evidence of a number of genetic mutations [11-13] and similar disease processes [14-16], such as hyperphosphorylation of a microtubule-associated protein in the brain, mediating both schizophrenia and dementia. Others advocate that a possible association between schizophrenia and dementia would not be ascribed to common etiology, but rather to a by-product of the higher rates of chronic medical conditions, substance abuse, and unhealthy lifestyle among individuals with schizophrenia compared to the general population.

Addressing several of the aforementioned concerns, several studies have attempted to appraise the hypothesis of a shared etiology between mental disorders, particularly schizophrenia, and dementia by determining mental disorders as risk factors for dementia. Zilkens et al. [17], in a case-control study, indicated that severe mental disorders in midlife were associated with a subsequent incidence of dementia, with schizophrenia ranging from a 2 -fold to a 12-fold higher risk of developing dementia, depending on age at onset. Analogous findings were presented by Ribe et al. [18], in a prospective cohort study, showing that subjects who suffered from schizophrenia experienced a more than 2-fold higher risk of being diagnosed with dementia. A review [19] of 20 longitudinal studies using cognitive screening tools or neuropsychological tests to examine the risk of cognitive decline or dementia in persons with schizophrenia found inconsistent results, but still with slightly more studies suggesting an increased risk. Nevertheless, even though the breadth of evidence insinuates that schizophrenia is a risk factor for dementia, none of the studies have been able to efficiently embody all information on somatic comorbidity, substance abuse, or adverse health risk factors, leaving the relevance of schizophrenia in the pathogenesis of dementia controversial.

To elucidate the relationship between schizophrenia and dementia, other studies have addressed the question of familial coaggregation between the two disorders. These studies have yielded incongruent results, with 2 case-control studies $[11,20]$ finding no co-occurrence of schizophrenia and dementia (except frontotemporal dementia), and 3 case-control studies [21-23] finding increased incidence of mental disorders and psychoses in first-degree relatives of dementia probands. These studies are characterized by a limited number of cases, missing contemplation of dementia as a heterogeneous syndromic entity, and the use of a case-control design, which may have led to a considerable recall bias regarding psychiatric history, making it difficult to distill the findings and to draw any substantial conclusions. 
Thus, because of the inadequate understanding of the observed association between schizophrenia and dementia, the present limitations in studies investigating familial coaggregation and the lack of large-scale epidemiological follow-up studies, we conducted a population-based study. We used administrative data to investigate familial coaggregation of schizophrenia and dementia. By generating a cohort with a sufficient sample size, follow-up time and sufficient induction period for the cohort to display a clinically significant risk of dementia, we aimed to pursue the following questions: (1) Does an offspring with schizophrenia increase the risk of Alzheimer's dementia, and is any elevated risk confounded or modified by somatic comorbidity or the individual's own mental disorders? (2) Is the elevated risk specific for offspring with schizophrenia? (3) Are the results changed if the analyses are repeated according to the individuals' own mental disorder status?

\section{Methods}

\section{Registers}

The Danish Civil Registration Register (DCRS) [24] was established in 1968 and contains information on all individuals who have lived in Denmark. The DCRS utilizes a civil person register number, a unique personal identification number, which enables accurate linkage between all administrative national registers and ensures that the entire population can be followed over time. The Danish Psychiatric Central Research Register (DPCR) [25] was established in 1969 as an electronic database. The DPCR contains information on all psychiatric admissions since 1969 and all outpatient contacts after 1994. There are no private psychiatric hospitals in Denmark. The Danish National Patient Register (DNPR) [26] was computerized in 1977. The DNPR contains information about every person in contact with the Danish hospital services. In 1994, the DNPR was expanded to include outpatient and emergency room contacts as well. Until 1993, the diagnostic system used in the Danish registers was the Danish version of the International Classification of Diseases, Eighth Revision (ICD-8) [27]. From 1994, the Danish version of the ICD-10 [28] was used.

\section{Study Population}

We used the DCRS [24] to identify all Danish residents born between January 1,1930, and December 31, 1953, and who were still residing in Denmark in 1968. The study cohort was restricted to include all individuals with children living in Denmark on their 50th birthday. In addition, the DCRS [24] was used to identify the children of the cohort. The study design was an epidemiological register-based prospective cohort study. The members of the cohort were followed from their 50th birthday until the date of the outcome, emigration, death, or end of the study (December 31, 2013), whichever came first.

\section{Main Outcome Measure and Definition}

Diagnoses of dementia were obtained from the DPCR [25] and the DNPR [26]. Diagnoses of the main outcome Alzheimer's dementia were obtained (ICD-8: 290.10; ICD-10: F00.0-9 and G30.0-9). The date of dementia onset was defined as the first day of hospital contact with a diagnosis of dementia in either the DPCR [25] or the DNPR [26]. All analyses were also performed for vascular dementia (ICD-8: 293.09-19; ICD-10: F01.0-9) and unspecified dementia (ICD-8: 290.09, 290.18-19; ICD-10: F03.9).

\section{Main Exposure}

The main exposure was offspring diagnosed with schizophrenia or a severe related psychotic episode during an admission or outpatient contact with a psychiatric ward. The 
Rohde et al.: Does Schizophrenia in Offspring Increase the Risk of Developing Alzheimer's Dementia?

Table 1. Basic characteristics of the study cohort stratified by whether or not the offspring had a psychiatric service contact

\begin{tabular}{|c|c|c|}
\hline \multirow[t]{2}{*}{ Characteristics of cohort } & \multicolumn{2}{|c|}{ Mental history in offspring } \\
\hline & $\begin{array}{l}\text { psychiatric contact in } \\
\text { offspring }\end{array}$ & $\begin{array}{l}\text { no psychiatric contact in } \\
\text { offspring }\end{array}$ \\
\hline Number of individuals & 278,811 & $1,117,985$ \\
\hline Person-years followed & $2,923,118.36$ & $22,224,723.71$ \\
\hline Women & $140,547(50.41 \%)$ & $567,932(50.80 \%)$ \\
\hline Mean number of children & 2.62 & 2.20 \\
\hline Individuals with Alzheimer's dementia & 2,282 & 10,208 \\
\hline \multicolumn{3}{|l|}{ Birthplace } \\
\hline Capital & 65,924 & 221,777 \\
\hline Capital suburb & 10,876 & 38,243 \\
\hline Provincial cities ${ }^{\mathrm{a}}$ & 30,321 & 118,569 \\
\hline Provincial towns ${ }^{\mathrm{b}}$ & 71,321 & 290,551 \\
\hline Rural areas & 100,369 & 448,845 \\
\hline Psychiatric service contact & 44,801 & 93,761 \\
\hline Schizophrenia & 5,837 & 11,075 \\
\hline Myocardial infarction & 20,632 & 71,313 \\
\hline Congestive heart failure & 17,852 & 57,348 \\
\hline Peripheral vascular disease & 21,661 & 71,213 \\
\hline Cerebrovascular disease & 33,946 & 116,656 \\
\hline Diabetes mellitus 1 and 2 & 24,430 & 79,516 \\
\hline
\end{tabular}

a With more than 100,000 inhabitants. ${ }^{b}$ With more than 10,000 inhabitants.

diagnosis of schizophrenia or related psychosis was obtained from the DPCR [25] and was defined as the following ICD codes: ICD-8: 295.09-99, 296.89, 297.09-99, 298.39, 301.83; ICD-10: F20-F29. Diagnoses of other mental disorders in the offspring were also obtained from the DPCR [25] (online suppl. table 1; see www.karger.com/doi/10.1159/000448395 for all online suppl. material).

\section{Confounding Variables}

Information on confounding somatic comorbidity was obtained from the DNPR [26]. A comorbidity known to be a risk factor for dementia $[9,29,30]$ and associated with schizophrenia [31-34] was selected. This included myocardial infarction (ICD-8: 410; ICD-10: I21-23), congestive heart failure (ICD-8: 427.09-11, 427.19, 428.99, 782.49; ICD-10: I50.0-9, I11.0, I13.0, I13.2), peripheral vascular disease (ICD-8: 440-445; ICD-10: I70-74, I77), cerebrovascular disease (ICD-8: 430-438; ICD-10: I60-69, G45-46), and diabetes mellitus (ICD-8: 249.00, 249.06-7, 249.09, 250.00, 250.06-7, 250.09; ICD-10: E10.0, E10.1, E10.9, E11.0, E11.1, E11.9). Information on birthplace, sex, and birth date was obtained from the DCRS [24].

\section{Statistical Analysis}

Incidence rate ratios (IRRs) of Alzheimer's dementia were estimated by log-linear Poisson regression with the logarithm of the person-years as an offset variable using the GENMOD procedure in SAS version 9.3. Outcome measures were denoted as IRRs, comparing individuals with offspring with schizophrenia or other psychiatric contact with individuals with offspring without psychiatric contact. All IRRs were adjusted for calendar year, birthplace, age, sex, and its interaction with age. In subsequent analyses, the IRRs were adjusted for somatic comorbidity and for the individuals' mental disorders, respectively. 
Table 2. IRRs for Alzheimer's dementia in individuals with an offspring with schizophrenia, other psychiatric service contact, bipolar disorder, other affective disorder or personality disorder compared to individuals with an offspring without psychiatric service contact

\begin{tabular}{|c|c|c|c|c|c|}
\hline Offspring's mental history & Cases $^{\mathrm{a}}$ & Person-years & $\operatorname{IRR}(95 \% \mathrm{CI})^{\mathrm{b}}$ & $\operatorname{IRR}(95 \% \mathrm{CI})^{\mathrm{c}}$ & $\operatorname{IRR}(95 \% \mathrm{CI})^{\mathrm{d}}$ \\
\hline Schizophrenia & 417 & $523,179.86$ & $1.10(1.00-1.21)$ & $1.09(0.99-1.20)$ & $0.97(0.88-1.07)$ \\
\hline Bipolar disorder & 29 & $26,731.51$ & $1.12(0.76-1.57)$ & $1.09(0.74-1.54)$ & $0.99(0.67-1.39)$ \\
\hline Other affective disorder & 244 & $252,733.53$ & $1.18(1.04-1.34)$ & $1.16(1.02-1.31)$ & $1.07(0.94-1.22)$ \\
\hline Personality disorder & 167 & $221,764.22$ & $1.21(1.03-1.40)$ & $1.18(1.01-1.37)$ & $1.08(0.92-1.25)$ \\
\hline Other psychiatric contact & 1,425 & $1,898,709.24$ & $1.13(1.06-1.19)$ & $1.11(1.05-1.17)$ & $1.05(0.99-1.11)$ \\
\hline No psychiatric contact & 10,208 & $22,224,723.71$ & 1.00 (reference) & 1.00 (reference) & 1.00 (reference) \\
\hline
\end{tabular}

${ }^{a}$ Number of individuals with Alzheimer's dementia. ${ }^{\mathrm{b}}$ Adjusted for calendar year, place of birth, sex and its interaction with age. ${ }^{c}$ Further adjustment for somatic comorbidity. ${ }^{\mathrm{d}}$ Further adjustment for the individuals' mental disorders.

Age, calendar year, schizophrenia, or psychiatric contact in the offspring and a history of the individuals' mental disorders were treated as time-dependent variables, whereas all other variables were considered time independent. Age was categorized for 50- to 80-yearolds in 5-year age levels and for 81- to 100-year-olds in 10-year age levels. Calendar years were categorized in 1-year periods from 1968 to 2013.

In additional analyses, we stratified by the individuals' mental disorders, first calculating IRRs for individuals with a history of mental disorders and then IRRs for individuals without a history of mental disorders. IRRs and 95\% confidence intervals (CI) were calculated by loglikelihood estimation [35]. All analyses were also performed for vascular and unspecified dementia. The study was approved by the Danish Protection Agency and did not require ethical approval according to Danish laws.

\section{Results}

\section{Population Characteristics}

In the cohort $(n=1,396,796)$, a total of 12,490 individuals developed Alzheimer's dementia during the 25.1 million person-years of follow-up. 2,282 of the individuals with Alzheimer's dementia had an offspring with a psychiatric contact, 417 of whom had an offspring with a schizophrenia diagnosis. 10,208 of the individuals with Alzheimer's dementia had an offspring without a psychiatric contact. Regarding psychiatric and comorbid illnesses at the end of the study, individuals with offspring with a psychiatric contact exhibited a higher prevalence of schizophrenia (2.09 vs. $0.91 \%$ ), myocardial infarction (7.40 vs. $6.38 \%$ ), congestive heart failure (6.40 vs. $5.13 \%$ ), peripheral vascular disease (7.77 vs. $5.13 \%$ ), cerebrovascular disease (12.18 vs. $10.43 \%$ ), and diabetes ( 8.76 vs. $7.11 \%$ ) than did the individuals with offspring without psychiatric contact (table 1 ).

\section{Risk of Alzheimer's Dementia}

Table 2 displays incident cases of Alzheimer's dementia and person-years of follow-up according to the psychiatric diagnosis in the offspring. Individuals with an offspring with schizophrenia had a 1.10-fold (IRR, 1.10; 95\% CI, 1.00-1.21) increased risk of Alzheimer's dementia (table 2, fourth column), compared with the reference group, i.e. those with an offspring without psychiatric contact. Adjusting this estimate for somatic comorbidity only slightly attenuated the IRR (IRR, 1.09; 95\% CI, 0.99-1.20), whereas adjustment for the indi- 


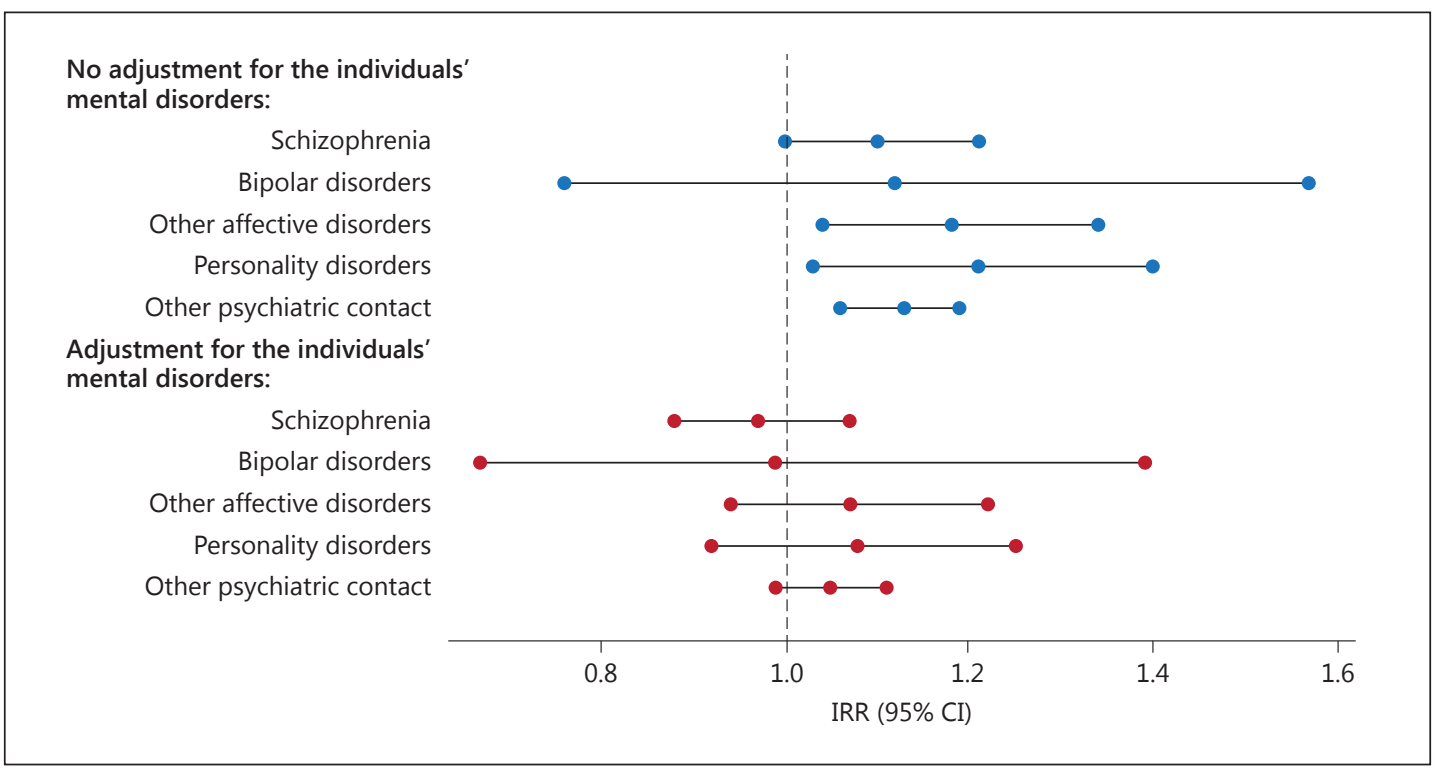

Fig. 1. IRRs for Alzheimer's dementia in individuals with an offspring with schizophrenia, other psychiatric service contact, bipolar disorder, other affective disorder or personality disorder compared to individuals with an offspring without psychiatric service contact.

viduals' own mental disorders diminished the significantly increased IRR (IRR, 0.97; 95\% CI, 0.88-1.07) (table 2, sixth column). Analogous findings were made for vascular and unspecified dementia (online suppl. tables 2 and 3 ).

When evaluating the subgroups characterized by age at Alzheimer's dementia onset, no association between offspring with schizophrenia and Alzheimer's dementia was found in any of the subgroups after adjustment for the individuals' own mental disorders, with IRR (IRR, 1.10; 95\% CI, 0.91-1.31) for individuals with Alzheimer's dementia before or equal to the age of 65 and IRR (IRR, 0.92; 95\% CI, 0.88-1.07) for individuals with Alzheimer's dementia above the age of 65 (data not shown).

Figure 1 shows the IRRs for Alzheimer's dementia according to the different psychiatric diagnoses in the offspring and how they are affected by the adjustment for the individuals' own mental disorders. All figures were compared with the reference group, i.e. those with offspring without psychiatric contact. The increased risk of Alzheimer's dementia was not specific to those having an offspring with schizophrenia, but its magnitude resembled the effect for individuals with offspring with other affective disorders, personality disorder, and other psychiatric contact (except bipolar disorder). As indicated in figure 1, the significantly increased IRRs for the different psychiatric diagnoses in the offspring were all diminished after adjustment for the individuals' own mental disorders. Furthermore, we analyzed whether any possible dose-response effect was observed, i.e. if the risk of Alzheimer's dementia differs according to the number of offspring with a psychiatric contact (data not shown). After adjustment for the individuals' own mental disorders, we did find a 1.16-fold (IRR, 1.16; 95\% CI, 1.04-1.29) increased risk of Alzheimer's dementia for individuals with 2 offspring with a psychiatric contact. We did not find any increased risk of Alzheimer's dementia after adjustment for the individuals' own mental disorders for individuals with 3 offspring with a psychiatric contact (IRR, 1.14; 95\% CI, 0.86-1.46).

Table 3 presents the partition of the cohort. To explore potential effect measure modification (interaction) of the individuals' own mental disorders, the analyses were stratified 
Rohde et al.: Does Schizophrenia in Offspring Increase the Risk of Developing Alzheimer's Dementia?

Table 3. IRRs for Alzheimer's dementia in individuals with an offspring with schizophrenia, other psychiatric service contact, bipolar disorder, other affective disorder or personality disorder compared to individuals with an offspring without psychiatric service contact

\begin{tabular}{lrl}
\hline Offspring's mental history & Cases $^{\mathrm{a}}$ & ${\text { IRR }(95 \% \mathrm{CI})^{\mathrm{b}}}$ \\
\hline Only individuals with mental disorders & & \\
$\quad$ Schizophrenia & 103 & $0.71(0.58-0.86)$ \\
Bipolar disorder & $7^{\mathrm{c}}$ & $0.72(0.31-1.40)$ \\
Other affective disorder & 70 & $1.05(0.82-1.32)$ \\
Personality disorder & 40 & $0.80(0.57-1.07)$ \\
Other psychiatric contact & 293 & $0.82(0.72-0.93)$ \\
No psychiatric contact & 1,628 & $1.00($ reference) \\
Only individuals with no mental disorders & & \\
Schizophrenia & 314 & $1.09(0.97-1.21)$ \\
Bipolar disorder & 22 & $1.10(0.70-1.63)$ \\
Other affective disorder & 174 & $1.07(0.92-1.24)$ \\
Personality disorder & 127 & $1.19(1.00-1.42)$ \\
Other psychiatric contact & 1,132 & $1.12(1.05-1.19)$ \\
No psychiatric contact & 8,580 & $1.00($ reference) \\
\hline
\end{tabular}

${ }^{a}$ Number of individuals with Alzheimer's dementia. ${ }^{\mathrm{b}}$ Adjusted for calendar year, birth place, sex and its interaction with age. ${ }^{\mathrm{c}}$ Should be interpreted with caution because of the few exposed cases.

according to the individuals' own mental disorder status. An offspring with schizophrenia did not have an increased risk of Alzheimer's dementia in either individuals with a history of mental disorders or individuals without a history of mental disorders. In addition, we were able to replicate the earlier findings of increased risk of all-cause dementia for individuals with schizophrenia. After adjustment for somatic comorbidity, substance and alcohol abuse, we found a 3.45-fold increased risk of all-cause dementia (IRR, 3.45; 95\% CI, 3.25-3.65) for individuals with a diagnosis of schizophrenia.

\section{Discussion}

\section{Main Findings}

To the best of our knowledge, this is the first population-based cohort study that investigates the association between the risk of Alzheimer's dementia and offspring with a schizophrenia diagnosis. We found little evidence of an increased risk of Alzheimer's dementia among subjects whose offspring had been diagnosed with schizophrenia, bipolar disorder, other affective disorders, personality disorder, or any other severe mental disorder. The increased risk of Alzheimer's dementia among individuals with an offspring with schizophrenia was diminished and not statistically significant when taking the individuals' own mental disorders into account. Analogous findings were made for offspring with other affective disorders, personality disorder, and any other severe mental disorder.

\section{Familial Coaggregation of Schizophrenia and Dementia - Shared Etiology?}

To date, only a few studies have addressed the question of familial comorbidity in schizophrenia and dementia, and they have generated opposing findings. Our findings in this study were consistent with the prior findings of no coaggregation between schizophrenia and Alzheimer's dementia from the larger case-control studies [11, 20], but they were in discrepancy with the finding of coaggregation between psychosis or mental disorders and Alzheimer's dementia in other case-control studies [21, 23]. However, the latter studies were confined by very small sample sizes, making the associations found debatable. A 
prevailing problem with studies in this area is the missing contemplation of Alzheimer's dementia as a highly heterogeneous disorder. Even though memory deficiency is the core symptom, the genotype and clinical phenotype are heterogeneous, with especially striking disparities between early-onset Alzheimer's dementia (EOAD) and late-onset Alzheimer's dementia (LOAD). Despite little neuropathological differences, EOAD, with an age at onset before the age of 65 years, is characterized by disease aggregation within families and the presence of specific mutations in some cases [36], whereas LOAD tends to be sporadic. This implies that if a shared etiology between schizophrenia and Alzheimer's dementia existed, a more pronounced association might be anticipated between schizophrenia and EOAD. Interestingly, none of the earlier studies in this area took this complexity and age-related dichotomy of Alzheimer's dementia into consideration, and an actual association between schizophrenia and EOAD could be diluted by the excess cases of LOAD. To our knowledge, only one earlier study has measured differences in the prevalence of a family history of mental disorders between EOAD and LOAD probands [37]. This case-control study revealed a more than 2-fold increase in a family history of mental disorders among EOAD probands compared to LOAD probands. The authors concluded that a possible explanation for this finding could be shared etiology between EOAD and some subtypes of mental disorders. This finding was in discrepancy with our finding of no increased risk of either EOAD or LOAD for individuals with an offspring with schizophrenia. However, their study was probably prone to recall bias, which might have resulted in false-positive diagnoses of Alzheimer's dementia as mental disorder among relatives of EOAD probands, leading to differential misclassification. In light of this, the present findings from our nationwide population-based study and the previous findings from the larger case-control studies in this area suggest that a family history of schizophrenia does not seem to increase the risk of Alzheimer's dementia, which may indicate that no shared etiology between the disorders exists. This is also reinforced by the fact that no estimates found in this study were characteristic for individuals with an offspring with schizophrenia, but that they were almost identical in magnitude for individuals with offspring with other affective disorders, personality disorder, and other psychiatric contact.

\section{Increased Risk of Dementia in Individuals with Schizophrenia - Do We Have an}

\section{Explanation?}

The finding of no familial coaggregation between schizophrenia and Alzheimer's dementia is conflicting with the growing body of literature emerging of an intriguing relationship between schizophrenia in the individual and the development of dementia [17-19]. However, whereas most of the studies investigating coaggregation used Alzheimer's dementia as the main outcome measure, the studies investigating schizophrenia in the individuals themselves used all-cause dementia (including subtypes such as vascular- and unspecified dementia).

There might be two explanations for the finding of an increased risk of dementia in subjects with schizophrenia. First, the higher risk of all-cause dementia could reflect a common underlying etiology for schizophrenia and some subtypes of dementia, which is a hypothesis that has been suggested [38]. Alternatively, the higher risk might be explained as being due to the fact that mental disorders, especially schizophrenia, could be a proxy variable for social adversity, chronic medical or poor living conditions, including low educational level and adverse health risk factors (tobacco smoking, alcohol assumption, low psychical activity, substance abuse, etc.) [39-42], which is an array of well-established risk factors for dementia $[9,29]$. As all-cause dementia includes dementia subtypes more prone to lifestyle patterns, such as vascular dementia [43], the association may reflect that individuals with mental disorders separate them in a number of ways compared to individuals without mental disorders regarding lifestyle patterns, which could make them more susceptible to develop 
dementia. This latter conception is in agreement with previous reports, in which several mental disorders have consistently been found to be associated with a shortened life expectancy, which may partly be explained by increased prevalence of somatic comorbidity and adverse health risk factors [44-46]. Notably, individuals with schizophrenia have been found to have a higher rate of mortality from natural causes compared with those with other mental disorders and the general population [45].

To account for this possible unhealthy lifestyle among individuals with schizophrenia, most of the studies investigating whether individuals with schizophrenia had an increased risk of dementia tried to adjust their findings for somatic comorbidity and substance abuse. However, the excess risk accounted for by these factors could be underestimated as somatic comorbidity and substance abuse are underdiagnosed and undertreated among individuals with schizophrenia [31,47]. This is probably caused by the fact that individuals with mental disorders often have poor medical monitoring, possibly exacerbated in schizophrenia by poor social communication skills and altered perception, rendering them less likely to seek medical care. Additionally, the studies were limited by the fact that information on somatic comorbidity and substance abuse was obtained from secondary health care registers, which do not contain information on conditions treated in the primary health care setting, and the fact that information on most adverse health risk factors were not obtained. This could indicate, that even though the studies tried to efficiently embody all information on somatic comorbidity and substance abuse, this may have been insufficient, meaning that the excess risk of dementia in individuals with schizophrenia might still be explained by underlying factors such as somatic comorbidity or adverse health risk factors.

\section{Schizophrenia and Dementia - What Do We Know Now?}

Even though individuals with schizophrenia have consistently been found to have an increased risk of dementia [17-19], it has long been debated whether this excess risk is due to a common etiology between the disorders or a by-product of the higher rates of somatic comorbidity and adverse health risk factors among individuals with schizophrenia [38]. Our findings of little coaggregation between schizophrenia and Alzheimer's dementia (as well as other subtypes of dementia), and the finding that this was not specific for schizophrenia but also seen for other mental disorder, may contribute to the debate by suggesting that no common etiology between schizophrenia and dementia exists. This finding might indicate that the excess risk of dementia in individuals with schizophrenia reflect that schizophrenia is a proxy variable for underlying risk factors for dementia. Whether these risk factors represent somatic comorbidity, adverse health risk factors, substance abuse or other risk factors among probands with schizophrenia is not known and cannot be extrapolated with our study.

\section{Strengths and Weaknesses}

The strength of this study lies in its epidemiological design. Using a prospective population-based design, information on psychiatric illnesses was collected from registers, thus avoiding the risk of selections and recall bias. Beyond this, the design provided a large study population with 25.1 million person-years of follow-up, making the study able to detect small effects. The selection bias due to nonparticipation was nonexistent, and loss to follow-up was negligible, as it only occurs when people die or leave the country. The latter is minimal due to low emigration and immigration rates in Denmark.

Our findings should also be interpreted in the light of limitations. First, our study results may have been underpowered to find an association, but this study is based on the entire Danish population and includes all patients given a clinical diagnosis of Alzheimer's dementia or schizophrenia, and similar data can only be obtained in very few countries. Second, diag- 
noses from the registers were used and may lack the reliability that sometimes can be obtained with structured diagnostic interviews. However, the diagnostic validity of an Alzheimer's dementia diagnosis was good, with $81 \%$ valid diagnoses [48]. Regarding the psychiatric diagnoses, a schizophrenia diagnosis had a high validity [49], which also applies to bipolar and other affective disorders [50], which minimized the risk of misclassification. However, by using the registers, we may have missed some information. Firstly, the Psychiatric Central Research Register was first established in 1969, and any information about psychiatric history before this year is not recorded. Secondly, depression and some other psychiatric disorders are often treated in the primary health care settings, which the registers do not contain information about. We may therefore have misclassified some individuals as nonpsychiatric individuals, which may have resulted in nondifferential misclassification and thereby tended to bias any of the risk estimates toward unity. With regard to dementia, approximately only two thirds of all cases of dementia in Denmark are diagnosed within the secondary health care [51], and we therefore accepted the milder types of Alzheimer's dementia to be missing. This is in agreement with the observation that $91 \%$ of the registered dementia diagnoses were moderate to severe dementia according to the ICD-10 criteria [48]. Thus, the interpretation of the results should be done with the notion that we have examined the most severe cases of Alzheimer's dementia.

As it is well known that mental disorders, especially schizophrenia, aggregate within families [52], a fundamental consideration was whether an offspring with schizophrenia should be conceptualized as an independent risk factor for Alzheimer's dementia or an intermediate factor on the pathway between schizophrenia in the individual and Alzheimer's dementia and thereby regarded as a proxy variable for schizophrenia in the individual. A great advantage of the latter would be that individuals in the study would be old enough to develop dementia and the offspring would be young enough to develop schizophrenia. This has especially been problematic in earlier studies investigating the risk of dementia in individuals with schizophrenia, as schizophrenia develops in early adolescence and dementia in late life, which requires registers with many years of observation. These studies $[17,19,53$, 54] have therefore only comprehended individuals developing schizophrenia at an old age and followed them for an insufficient duration of time for them to develop all types of dementia, making it difficult to generalize their findings. However, as over two thirds of cases of schizophrenia occur sporadically [52], regarding an offspring with schizophrenia as a proxy variable would be misleading in the majority of cases. It was therefore more obvious to regard an offspring with schizophrenia itself as a risk factor for dementia, which made it possible to comment on familial coaggregation between the disorders and thereby speculate about common etiology. However, we acknowledge that families to some degree might share the environment, resulting in an unhealthier lifestyle among individuals with an offspring with schizophrenia compared to individuals with an offspring without psychiatric contact. This means that complex gene-environment correlations may influence the association. However, in contrast to earlier studies investigating dementia in individuals with schizophrenia, this approach will most likely exclude more of the effect ascribed to environmental factors.

\section{Implications}

In conclusion, this population-based study found little evidence for an association between an offspring with schizophrenia and subsequent risk of Alzheimer's dementia (as well as other subtypes of dementia), which may suggest that a common etiology between schizophrenia and dementia is less likely. These findings make an important contribution to the literature by implying that the trajectory towards dementia among individuals with schizophrenia is not irreversible and may be prevented by efficient prevention strategies and 
medical care for individuals with schizophrenia. Identification of specific factors that underlie the excess risk of dementia in individuals with schizophrenia, whether it is a somatic comorbidity, an adverse health risk factor, or unknown risk factors, may therefore be of relevance in the prevention of dementia in this high-risk population [9].

\section{Acknowledgements}

This study was supported by the Lundbeck Foundation. The funder had no role in the study design, execution or analysis, or the manuscript preparation.

\section{Disclosure Statement}

The authors declare that they have no conflicts of interest concerning this article.

\section{References}

1 van der Flier WM, Scheltens P: Epidemiology and risk factors of dementia. J Neurol Neurosurg Psychiatry 2005;76(suppl 5):v2-v7.

-2 Nilsson K, Gustafson L, Hultberg B: Survival in a large elderly population of patients with dementia and other forms of psychogeriatric diseases. Dement Geriatr Cogn Disord 2011;32:342-350.

-3 Ferri CP, Prince M, Brayne C, Brodaty H, Fratiglioni L, Ganguli M, Hall K, Hasegawa K, Hendrie H, Huang Y, Jorm A, Mathers C, Menezes PR, Rimmer E, Scazufca M: Global prevalence of dementia: a Delphi consensus study. Lancet 2005;366:2112-2117.

4 Hebert LE, Weuve J, Scherr PA, Evans DA: Alzheimer disease in the United States (2010-2050) estimated using the 2010 census. Neurology 2013;80:1778-1783.

5 Alzheimer's Disease International: Policy Brief for Heads of Government: The Global Impact of Dementia 2013-2050. London, Alzheimer's Disease International, 2013.

-6 Schaller S, Mauskopf J, Kriza C, Wahlster P, Kolominsky-Rabas PL: The main cost drivers in dementia: a systematic review. Int J Geriatr Psychiatry 2015;30:111-129.

7 Raz L, Knoefel J, Bhaskar K: The neuropathology and cerebrovascular mechanisms of dementia. J Cereb Blood Flow 2016;36:172-186.

8 Broadstock M, Ballard C, Corbett A: Latest treatment options for Alzheimer's disease, Parkinson's disease dementia and dementia with Lewy bodies. Expert Opin Pharmacother 2014;15:1797-1810.

-9 Deckers K, van Boxtel MP, Schiepers OJ, de Vugt M, Munoz Sanchez JL, Anstey KJ, Brayne C, Dartigues JF, Engedal K, Kivipelto M, Ritchie K, Starr JM, Yaffe K, Irving K, Verhey FR, Kohler S: Target risk factors for dementia prevention: a systematic review and Delphi consensus study on the evidence from observational studies. Int J Geriatr Psychiatry 2015;30:234-246.

10 Bae JB, Kim YJ, Han JW, Kim TH, Park JH, Lee SB, Lee JJ, Jeong HG, Kim JL, Jhoo JH, Yoon JC, Kim KW: Incidence of and risk factors for Alzheimer's disease and mild cognitive impairment in Korean elderly. Dement Geriatr Cogn Disord 2015;39:105-115.

-11 Schoder D, Hannequin D, Martinaud O, Opolczynski G, Guyant-Marechal L, Le Ber I, Campion D: Morbid risk for schizophrenia in first-degree relatives of people with frontotemporal dementia. Br J Psychiatry 2010;197: 28-35.

12 Cooper JJ, Ovsiew F: The relationship between schizophrenia and frontotemporal dementia. J Geriatr Psychiatry Neurol 2013;26:131-137.

-13 Momeni P, DeTucci K, Straub RE, Weinberger DR, Davies P, Grafman J, Hardy J, Huey ED: Progranulin (GRN) in two siblings of a Latino family and in other patients with schizophrenia. Neurocase 2010;16:273-279.

14 Deutsch SI, Rosse RB, Lakshman RM: Dysregulation of tau phosphorylation is a hypothesized point of convergence in the pathogenesis of Alzheimer's disease, frontotemporal dementia and schizophrenia with therapeutic implications. Prog Neuropsychopharmacol Biol Psychiatry 2006;30:1369-1380.

-15 Deutsch SI, Rosse RB, Deutsch LH: Faulty regulation of tau phosphorylation by the reelin signal transduction pathway is a potential mechanism of pathogenesis and therapeutic target in Alzheimer's disease. Eur Neuropsychopharmacol 2006;16:547-551.

16 Guidotti A, Auta J, Davis JM, Di-Giorgi-Gerevini V, Dwivedi Y, Grayson DR, Impagnatiello F, Pandey G, Pesold C, Sharma R, Uzunov D, Costa E: Decrease in reelin and glutamic acid decarboxylase67 (GAD67) expression in schizophrenia and bipolar disorder: a postmortem brain study. Arch Gen Psychiatry 2000;57:1061-1069. 
Zilkens RR, Bruce DG, Duke J, Spilsbury K, Semmens JB: Severe psychiatric disorders in mid-life and risk of dementia in late life (age 65-84 years): a population based case-control study. Curr Alzheimer Res 2014;11: 681-693.

18 Ribe AR, Laursen TM, Charles M, Katon W, Fenger-Gron M, Davydow D, Chwastiak L, Cerimele JM, Vestergaard M: Long-term risk of dementia in persons with schizophrenia: a Danish population-based cohort study. JAMA Psychiatry 2015;72:1095-1101.

19 Shah JN, Qureshi SU, Jawaid A, Schulz PE: Is there evidence for late cognitive decline in chronic schizophrenia? Psychiatr Q 2012;83:127-144.

20 Heun R, Kockler M, Ptok U: Psychiatric disorders in relatives of subjects with Alzheimer's disease. No evidence for common genetic risk factors. Eur Arch Psychiatry Clin Neurosci 2002;252:93-97.

-21 Martin RL, Gerteis G, Gabrielli WF Jr: A family-genetic study of dementia of Alzheimer type. Arch Gen Psychiatry 1988;45:894-900.

22 Li G, Shen YC, Li YT, Chen CH, Zhau YW, Silverman JM: A case-control study of Alzheimer's disease in China. Neurology 1992;42:1481-1488.

23 Narayanaswamy JC, Varghese M, Jain S, Sivakumar PT, Prakash O, Bharath S, Kandavel T: Is there a familial overlap between dementia and other psychiatric disorders? Int Psychogeriatr 2011;23:749-755.

24 Pedersen CB: The Danish Civil Registration System. Scand J Public Health 2011;39:22-25.

25 Mors 0, Perto GP, Mortensen PB: The Danish Psychiatric Central Research Register. Scand J Public Health 2011;39:54-57.

26 Lynge E, Sandegaard JL, Rebolj M: The Danish National Patient Register. Scand J Public Health 2011;39:30-33.

27 World Health Organization: Classification of diseases: Extended Danish-Latin version of the World Health Organization International Classification of Diseases, 8th revision, 1965 (in Danish). Copenhagen, Danish National Board of Health, 1971.

28 World Health Organization: WHO ICD-10: International Statistical Classification of Diseases and Health Related Problems (in Danish). Copenhagen, Munksgaard Danmark, 1993.

29 McCullagh CD CD, McIlroy SP, Passmore AP: Risk factors for dementia. Adv Psychiatr Treat 2001;7:24-31.

-30 Butters MA, Young JB, Lopez O, Aizenstein HJ, Mulsant BH, Reynolds CF 3rd, DeKosky ST, Becker JT: Pathways linking late-life depression to persistent cognitive impairment and dementia. Dialogues Clin Neurosci 2008; 10:345-357.

-31 Laursen TM, Munk-Olsen T, Agerbo E, Gasse C, Mortensen PB: Somatic hospital contacts, invasive cardiac procedures, and mortality from heart disease in patients with severe mental disorder. Arch Gen Psychiatry 2009;66:713-720.

32 Fan Z, Wu Y, Shen J, Ji T, Zhan R: Schizophrenia and the risk of cardiovascular diseases: a meta-analysis of thirteen cohort studies. J Psychiatr Res 2013;47:1549-1556.

-33 Eaton WW, Byrne M, Ewald H, Mors O, Chen CY, Agerbo E, Mortensen PB: Association of schizophrenia and autoimmune diseases: linkage of Danish national registers. Am J Psychiatry 2006;163:521-528.

-34 Gough SC, O'Donovan MC: Clustering of metabolic comorbidity in schizophrenia: a genetic contribution? J Psychopharmacol (Oxford) 2005;19:47-55.

35 Clayton D, Hills M: Statistical Models in Epidemiology. New York, Oxford University Press, 1993.

-36 Rademakers R, Cruts M, Van Broeckhoven C: Genetics of early-onset Alzheimer dementia. Sci World J 2003;3: 497-519.

-37 Devi G, Williamson J, Massoud F, Anderson K, Stern Y, Devanand DP, Mayeux R: A comparison of family history of psychiatric disorders among patients with early- and late-onset Alzheimer's disease. J Neuropsychiatry Clin Neurosci 2004;16:57-62.

-38 Lyketsos CG, Peters ME: Dementia in patients with schizophrenia: evidence for heterogeneity. JAMA Psychiatry 2015;72:1075-1076.

39 Buckley PF, Miller BJ, Lehrer DS, Castle DJ: Psychiatric comorbidities and schizophrenia. Schizophr Bull 2009; 35:383-402.

-40 Manzanares N, Monseny R, Ortega L, Montalvo I, Franch J, Gutierrez-Zotes A, Reynolds RM, Walker BR, Vilella E, Labad J: Unhealthy lifestyle in early psychoses: the role of life stress and the hypothalamic-pituitary-adrenal axis. Psychoneuroendocrinology 2014;39:1-10.

41 Brown S, Birtwistle J, Roe L, Thompson C: The unhealthy lifestyle of people with schizophrenia. Psychol Med 1999;29:697-701.

42 Leucht S, Burkard T, Henderson J, Maj M, Sartorius N: Physical illness and schizophrenia: a review of the literature. Acta Psychiatr Scand 2007;116:317-333.

43 O’Brien JT, Thomas A: Vascular dementia. Lancet 2015;386:1698-1706.

-44 Eaton WW, Martins SS, Nestadt G, Bienvenu OJ, Clarke D, Alexandre P: The burden of mental disorders. Epidemiol Rev 2008;30:1-14.

45 Laursen TM, Munk-Olsen T, Nordentoft M, Mortensen PB: Increased mortality among patients admitted with major psychiatric disorders: a register-based study comparing mortality in unipolar depressive disorder, bipolar affective disorder, schizoaffective disorder, and schizophrenia. J Clinl Psychiatry 2007;68:899-907.

46 Oud MJ, Meyboom-de Jong B: Somatic diseases in patients with schizophrenia in general practice: their prevalence and health care. BMC Fam Pract 2009;10:32. 
47 Smith DJ, Langan J, McLean G, Guthrie B, Mercer SW: Schizophrenia is associated with excess multiple physicalhealth comorbidities but low levels of recorded cardiovascular disease in primary care: cross-sectional study. BMJ Open 2013;3:e002808.

-48 Phung TK, Andersen BB, Hogh P, Kessing LV, Mortensen PB, Waldemar G: Validity of dementia diagnoses in the Danish hospital registers. Dement Geriatr Cogn Disord 2007;24:220-228.

49 Uggerby P, Ostergaard SD, Roge R, Correll CU, Nielsen J: The validity of the schizophrenia diagnosis in the Danish Psychiatric Central Research Register is good. Dan Med J 2013;60:A4578.

50 Kessing L: Validity of diagnoses and other clinical register data in patients with affective disorder. Eur Psychiatry 1998;13:392-398.

51 Phung TK, Waltoft BL, Kessing LV, Mortensen PB, Waldemar G: Time trend in diagnosing dementia in secondary care. Dement Geriatr Cogn Disord 2010;29:146-153.

52 Tandon R, Keshavan MS, Nasrallah HA: Schizophrenia, 'just the facts' what we know in 2008. 2. Epidemiology and etiology. Schizophr Res 2008;102:1-18.

53 Korner A, Lopez AG, Lauritzen L, Andersen PK, Kessing LV: Late and very-late first-contact schizophrenia and the risk of dementia - a nationwide register based study. Intl J Geriatr Psychiatry 2009;24:61-67.

54 Brodaty H, Sachdev P, Koschera A, Monk D, Cullen B: Long-term outcome of late-onset schizophrenia: 5-year follow-up study. Br J Psychiatry 2003;183:213-219. 